\title{
EVALUATING A NATURAL HORSEMANSHIP PROGRAM IN RELATION TO THE ISES FIRST PRINCIPLES OF HORSE TRAINING
}

S. North ${ }^{1}$, A. Hemingway ${ }^{2,},{ }^{*}$ A. McLean ${ }^{3}$, H. Laurie ${ }^{4}$ and C. Ellis-Hill ${ }^{2}$

${ }^{1}$ Mixed Reality Laboratory, School of Computer Science, Jubilee Campus, University of Nottingham, Nottingham NG8 1BB, UK

${ }^{2}$ Bournemouth House B129, Bournemouth University, 19 Christchurch Road, Bournemouth, BH1 3LH, UK

${ }^{3}$ AEBC (Australian Equine Behaviour Centre), 730 Clonbinane Road, Clonbinane, Victoria, Australia, 3658

${ }^{4}$ TheHorseCourse, PO Box 6500, Dorchester, Dorset, DT1 9BQ, UK

*Corresponding author: aheming@bournemouth.ac.uk

The ISES training principles provide an excellent starting point for professionals and horse owners. Currently, there does not seem to be an accepted protocol for evaluating horse training programs against the ISES principles. We suggest an approach to this, using Parelli Natural Horsemanship as our subject for evaluation. This initial pilot study (single-subject / $n=1$ ), trials two analytical methods, as applied to the current, video-based teaching materials from Parelli (latest 2015 DVD set). The two methods used were: (i) ethology-based video observation / logging and (ii) discourse analysis of the language used to teach. The ethology-based approach logged observed frequencies of the ISES principles. Inter Observer Reliability was assessed using ICC (Intra Class Correlation). Discourse analysis considered both the context and meaning of training language, to both the speaker and audience. Ethology-based results found all ISES principles present (1-10). High frequency counts for principles $2 \& 10$. Low counts for principles $5 \& 7$. Inter Observer Reliability ( 2 observers) was in the 'excellent' range (ICC=0.79). The high ICC value suggests that a minimal amount of measurement error was introduced by the independent observers, and therefore statistical power is not substantially reduced. At this stage (without an ICC value closer to 1.0 or further calibrating observers), increasing the evidence against random effects would require more extensive trials $(p=0.16)$. The interim results from the discourse analysis show consistent congruence between the Parelli materials and the ISES principles, particularly in principles: 1, 2, 7, 9 and 10.

Keywords: discourse analysis, ethogram, ethology, ISES training principles, Parelli. 